\title{
Headache and Health-Related Job Loss among Disadvantaged Women
}

\author{
Shawn M. Kneipp, Ph.D., RN, ANP-BC, APHN-BC [Associate Professor], \\ School of Nursing \\ Linda L. Beeber, Ph.D., RN, CS [Professor], and \\ School of Nursing
}

Laura A. Linnan, Sc.D., CHES [Professor]

Gillings School of Global Public Health

\author{
Keywords \\ womens health; headache; socioeconomic status; employment; health disparities
}

\section{Introduction}

From a public health perspective, the global burden of headache is significant. According to the World Health Organization (WHO), 46\% of adults suffer from headaches (Stovner et al., 2007; World Health Organization, 2012). The vast majority of headaches are benign, and are classified as primary headache disorders based on the International Classification of Headache Disorders-II (ICHD-II) (International Headache Society (IHS), 2005). This class of headache predominantly includes episodic and chronic forms of tension-type headache (TTH), migraine headache with and without aura, and cluster headache. In the adult population, the lifetime prevalence of TTH is $52 \%$, migraine headache $18 \%$, and cluster headache 1\% (Hainer \& Matheson, 2013; Jensen \& Stovner, 2008). Although TTHs may be more common, migraine headaches account for the vast majority of disability, and afflict women at nearly 3 times the rate as they do men - with $17.3 \%$ of women and $5.7 \%$ of men meeting the ICHD-II migraine criteria (Buse et al., 2013). Up to $72 \%$ of women who present with headache symptoms meet ICHD-II criteria for episodic, chronic, or probable migraine (Bigal, Bordini, \& Speciali, 2000). Primarily as a result of sex differences in migraine prevalence and its associated symptom burden, headache is one of the top five disorders responsible for disability among women worldwide (World Health Organization, 2012).

(C) 2014 Elsevier Inc. All rights reserved.

Corresponding Author: Shawn M. Kneipp, Ph.D., RN, ANP-BC, APHN-BC Associate Professor The University of North Carolina at Chapel Hill School of Nursing CB\#7460, Carrington Hall Chapel Hill, NC 27599 Office Phone: 919-966-5425 skneipp@email.unc.edu.

Publisher's Disclaimer: This is a PDF file of an unedited manuscript that has been accepted for publication. As a service to our customers we are providing this early version of the manuscript. The manuscript will undergo copyediting, typesetting, and review of the resulting proof before it is published in its final citable form. Please note that during the production process errors may be discovered which could affect the content, and all legal disclaimers that apply to the journal pertain. 
Headache-related disability also has a substantial adverse effect on workplace outcomes and costs. An estimated $31 \%$ of adults with migraine report losing at least one day of work in the prior three months, and are absent an average of 10.7 days per year due to their headache symptoms (Kessler, Shahly, Stang, \& Lane, 2010; Lipton, Stewart, Diamond, Diamond, \& Reed, 2001). Moreover, 51\% report their work productivity was reduced by at least 50\% (Lipton et al., 2001). The work absenteeism associated with migraine has been estimated to result in a loss of productivity of $\$ 1,165$ per individual, and up to $\$ 13$ billion dollars annually in the US (Kessler et al., 2010).

As with many other chronic disease states influenced by stress, behavior, and/or lifestyle factors, recent studies have found an inverse relationship between socioeconomic status (SES) and migraine prevalence (Buse et al., 2013; Stewart, Roy, \& Lipton, 2013). Using nationally representative data, Buse and colleagues (2013) found both women and men in the highest income group were $46 \%$ and $55 \%$ less likely to have migraine headaches than those in the lowest income bracket, respectively. Others have found lower income groups experience more frequent migraine episodes, more severe pain, and more disability from migraines (Stewart et al., 2013).

In isolation, the higher prevalence among and more severe symptom burden experienced by women who are socioeconomically disadvantaged (henceforth referred to as 'disadvantaged') can be viewed by health care providers as one of many conditions requiring appropriate diagnosis and treatment in order to improve a patients current quality of life. From a social, economic, and life course perspective, however, the potential impact of poorly-controlled symptoms over time can be significant. Disadvantaged women, for example, typically work in employment sectors which do not extend paid sick leave and may be less tolerant of illness-related absences (Singh-Manoux, Ferrie, Chandola, \& Marmot, 2004; Strully, 2009).

Given the level of disability associated with migraine and the characteristics of their employment conditions, headache disorders may exacerbate the pre-existing vulnerability of disadvantaged women to periods of recurrent unemployment and economic instability. As nurse practitioners providing primary care to this population, we need to better understand the unique risks that might be incurred by this group that could negatively impact their economic stability. Despite this, little is known about the impact of headache on employment among disadvantaged women, in particular. Therefore, the purpose of this study was to examine the relationships among headache as a chronic health condition (CHC), health care system and medication utilization patterns for headache, and employment history among disadvantaged women. More specifically, the aims of the study were to:

1. Describe (a) the frequency of self-reported headache, (b) health care utilization patterns related to headache, and (c) patterns of medication use for headache, and

2. Examine the (a) association between self-reported headache as a $\mathrm{CHC}$ and recent health-related job loss, and (b) assess the strength of this association relative to other high-prevalence chronic health conditions among a sample of SESdisadvantaged women. 


\section{Methods}

This study was conducted as a secondary analysis of existing data using a descriptive, multivariate correlational cross-sectional design. Approval was granted from two university Institutional Review Boards affiliated with the first author for both data collection and analysis.

\section{Sample}

The study uses baseline data from a recent randomized controlled trial (RCT) that tested a public health nursing case management intervention with 432 disadvantaged women in North Central Florida between 2005 and 2010. Details of the intervention development, RCT methods, and other health and employment-related study findings are reported elsewhere ([AUTHORS 2009, 2011, 2012). Women in the sample were all unemployed at baseline and had to either: (a) self-report having at least one health condition considered 'chronic' that had been diagnosed by a health provider, or (b) have met depression, general anxiety disorder, or post-traumatic stress disorder criteria using Structured Clinical Interview for DSM Disorders (SCID) modules (First, Spitzer, Gibbon, \& Williams, 2002). These criteria were selected given the potential for these conditions to interfere with physical, social, and/or emotional functioning. "Chronicity" was defined as "conditions that are generally not cured, once acquired" ([AUTHORS, 2011, p.1760]). Baseline sociodemographic sample characteristics are presented in Table 1. Women in this sample were predominantly Black (56.3\%), single (88.4\%), relatively young ( $x$ àge $=29.8$ years), and had a lower-level of education (60.2\% had a high school diploma/GED or less). All women enrolled in the study were receiving Medicaid at the time baseline data were collected.

\section{Measures}

Study participants provided self-report data as they completed a comprehensive healthrelated questionnaire. The questionnaire included health history-related questions commonly asked during a primary care visit, investigator-constructed questions, and a battery of standardized instruments to measure select health outcomes (i.e., the MOS Short-Form 12 Version 2, the Patient Health Questionnaire-9, among others).

The investigator-constructed items asked participants to select the chronic health conditions (CHCs) they had been diagnosed with by a health care provider from a comprehensive list. The presence of "headache" as a CHC was identified by women when completing this checklist, without further specification of headache type. For each $\mathrm{CHC}$ selected, they were asked to provide information on how long they had had the condition (in years), the number of emergency department (ED) visits made in the past year for the specified condition, and the medications taken for the specific condition. Headache medication use data were collected in narrative form and recoded by the study principal investigator into the following drug class categories: triptans, ergots, beta blockers, anticonvulsants, non-steroidal antiinflammatory drugs (NSAIDS), controlled substances, and other over-the-counter preparations. They were also asked to respond to the following item: "In the past year, how many jobs have you had to leave due to health?" 


\section{Data Analysis}

Data analyses were conducted using Stata/SE Version 13.0 (StataCorp LP, 2013).

Descriptive statistics were used to generate means, standard deviations, and frequencies for the sociodemographic, employment, and health condition characteristics and to meet Aim 1. To meet Aim 2, we applied chi-square statistics and logistic regression. We used a hierarchical regression approach to meet Aim 2a, we entered headache as the first independent variable into the model, followed sequentially by age, race, educational level, and the number of CHCs as covariates. To meet Aim 2b, a second logistic regression model was constructed using a forward, stepwise approach with the five most frequently-reported $\mathrm{CHCs}$ as independent variables. To ensure all CHCs even minimally associated with healthrelated job loss were represented in the final model, we set the significance criteria at 20 for model inclusion. We adjusted for age, race, and education level by locking these variables into the stepwise selection process. We did not, however, adjust for the number of CHCs in this analysis, as that would have introduced significant multicollinearity within the series of independent variables of interest incorporated in the model (i.e., the five most frequentlyoccurring CHCs). A post-estimation power analysis was conducted for the full Aim 2 regression model (StataCorp LP, 2013). Power was estimated using the probability of job loss with and without headache along with the intercorrelations among the independent variables in the full model, which ranged from .001 to .45 . Using these parameters, a sample size of 156 was sufficient to detect the main effects of variables on the full model at a power of .80. The final analytic sample to meet Aim 1 included all 432 women who completed data collection at baseline, with the final sample for Aim 2 including the 348 women who had been employed in the prior year (and hence could be considered "at risk" for job loss).

\section{Results}

Headache was the most frequently reported CHC, at $52.6 \%(\mathrm{n}=227)$. In rank order, the next four included back pain (49.8\%), depression (39.6\%), allergies (37.5\%), and anxiety (26.6\%). Of the 227 women that reported headache as a CHC, $36.6 \%(n=83)$ indicated that they made at least one ED visit in the prior year due to headache. The vast majority (54.7\%, $\mathrm{n}=123$ ) with headache were using over-the-counter (OTC) medications (including nonsteroidal anti-inflammatory drugs (NSAIDS), acetaminophen, and aspirin) to treat their symptoms. Nearly $30 \%(\mathrm{n}=67)$ were using no medications at all for headache, $7.6 \%(\mathrm{n}=17)$ were using triptans, and $8 \%(\mathrm{n}=18)$ reported taking opiates or scheduled narcotics. Among women who reported an ED visit for headache in the prior year, $91 \%$ reported their headaches had been present for two or more years. In addition, $83 \%$ who were using controlled substances for headache symptoms, $53 \%$ who were taking migraine-specific medications, and 34\% who were taking no medication at all for headache made at least one visit to the $\mathrm{ED}$ in the prior year.

Findings from the Aim 2 logistic regression analyses are presented in Table 2. The unadjusted odds of having left a job in the prior year due to health (henceforth referred to as "health-related job loss" for brevity) was nearly twice that for women who reported headache as a CHC compared to those who did not $(\mathrm{OR}=1.99, \mathrm{p}<.05)$. This association remained essentially unchanged as age, race, and education were sequentially added as covariates to the model. As noted in Model 5 (Table 2), when the number of CHCs was 
added to the model it became a statistically significant explanatory variable $(\mathrm{OR}=1.35, \mathrm{p}<$. $05)$, while the association between headache and health-related job loss was attenuated and was no longer statistically significant $(\mathrm{OR}=1.40)$. Also noted in Table 2 is the association between education and health-related job loss, whereby women with a high school diploma or GED were approximately half as likely to have reported health-related job loss as women with less than a high school diploma or GED (Models 4 and 5, OR $=.51$ and $.51, \mathrm{p}<.05$ for both).

To better understand the association between the number of CHCs, education, and healthrelated job loss, and examine how this may differ across women with and without headache, we conducted additional post-estimation analyses based on the initial regression findings. First, we simply examined the difference in the mean number of CHCs by job loss using an independent t-test, where women who had reported a health-related job loss in the prior year had a slightly higher number of CHCs $(x \equiv 4.7)$ and those that had not reported a healthrelated job loss $(x=3.3)(p<.001)$. We then (separately) included interaction term for (1) the number of $\mathrm{CHCs}$ and headache, and (2) education level and headache to the full regression model (Model 5, Table 2) while holding other covariates in the model constant (age, race, and education) (data not shown). The number of CHCs $\mathrm{x}$ headache interaction term was not significant $(\mathrm{OR}=.98, p=.90)$, indicating there was no difference in the direction or magnitude of the relationship between having a higher (or lower) number of CHCs and health-related job loss based on whether women reported headache. The education level $\times$ headache interaction was also not statistically significant ( $\mathrm{OR}=.78, p=.39)$, indicating there was no difference in the odds of having lost a job due to health in the prior year for women with headache across levels of education, while holding other covariates in the model constant.

Finally, to examine what the more nuanced relationship between headache, education, and number of CHCs on job loss, we also calculated the marginal effect of headache based on number of CHCs and education level, while adjusting for age and race (Mitchell, 2012). Given $93 \%$ of the sample had between one and seven CHCs, we estimated the marginal effect of headache for women at each $\mathrm{CHC}$ value between one and seven - first while holding education constant, and then by examining across the three education levels. Although the marginal effects were not statistically significant, as depicted in Figure 1, having headache as a $\mathrm{CHC}$ increases the probability of health-related job loss from $4.5 \%$ for women with one CHC to just over $8 \%$ for those with 6 or 7 CHCs. As depicted in Figure 2, although the marginal effects were also not statistically significant, there are some trends in the marginal probabilities of health-related job loss for women with headache that vary not only by the number of CHCs but also by level of education. Specifically, these marginal effect trends suggest headache accounts for a 6-8.5\% increased probability that women with less than a $12^{\text {th }}$ grade education had a health-related job loss if they had between 1 to 5 CHCs, while the probability for women with a high school diploma/GED and those with any type of college education or technical training is slightly less (3.5-7.5\%) if they had between 1 and 5 CHCs. These marginal effects increase as the number of CHCs increase across all groups, until they converge at $6 \mathrm{CHCs}$, where the probability is essentially the same for women regardless of education level (8\%). 
When the five most frequently occurring CHCs (headache, back pain, depression, allergies, and anxiety) were directly compared to one another as predictors of health-related job loss, only headache and allergies were significant ( $p=.004$ and .02 , respectively) while adjusting for age, race, and education. Back pain, depression, and anxiety were not significant independent predictors of health-related job loss, even though the significance criteria for inclusion in the model was set liberally $(\mathrm{p}=.20)$. This finding indicates that, across the five most prevalent disorders, headache is one of the CHCs most associated with job loss, as is having allergies. A headache $\times$ allergies interaction term added to the model indicated that women who reported both headache and allergies as CHCs were nearly three times as likely to have had a job loss compared to those who had neither headache nor allergies ( $\mathrm{OR}=2.9$, $\mathrm{p}=<.001$, data not shown).

\section{Study Limitations}

There are limitations of this study that should be noted. First, given the way in which CHC data were collected during the primary study, we are unable to discern which women in the sample with headache met diagnostic criteria for migraine as opposed to some other form of headache. In this sample, women identified whether they had headache as a chronic health condition that had been diagnosed by a provider, but without specificity regarding headache type. Based on the demographic and health-related composition of the study sample, it is reasonable to presume the vast majority were suffering from some form of a primary headache disorder. Given that: (1) at least one study found $72 \%$ of women presenting to primary care clinics with headache met diagnostic criteria for migraine (Bigal et al., 2000), and (2) the prevalence of migraine among lower-income women is 1.5 times that of women in higher income groups (20.6\% vs. $13.6 \%$, respectively) (Buse et al., 2013), it follows that a high proportion of women with headache in this study sample would also likely meet ICHDII diagnostic criteria for episodic, chronic, or probable migraine (International Headache Society (IHS), 2005). Although this likelihood is high, the lack of a definitive diagnosis makes comparing our findings to those of other studies - most of which relate to migraine tenuous. Second, while we had a large enough sample to detect the main effects in our regression models for Aim 2, it is possible that there was not sufficient power to detect the interactions and more nuanced marginal effect gradients that were explored post-hoc; thus, the inferences that can be drawn from the trends that were not statistically significant should be considered only trends, and require further exploration in a larger sample. Despite these limitations, the findings do make a novel contribution to our understanding of the extent to which headache is associated with recent health-related job loss among disadvantaged women.

\section{Discussion \& Recommendations}

Overall, these findings suggest that low income women who report headache as a CHC are twice as likely to have lost a job in the prior year due to health regardless of age, race, or education level. Two other factors played an important role in this relationship: a higher number of chronic health conditions, and having both headache and allergies as comorbid conditions. In the context of a greater number of $\mathrm{CHCs}$, headache becomes less relevant in explaining job loss, while having both headache and allergies increases the likelihood of job 
loss above and beyond having neither of these conditions. In addition, although nuanced, trends in our data suggest women with headache who also have less than a high school education or GED may be at a somewhat greater risk for job loss than those with more education when they have between 1 and 5 CHCs.

These findings are consistent with other findings in the literature. Ku and colleagues (2006), for example, found the prevalence of migraine was much higher among adults with allergic rhinitis (34\%) than in non-atopic, non-rhinitis controls (2\%). Recent study findings further suggest adults with both a diagnosis of migraine and rhinitis symptoms have greater headache days per month and headache-related disability than those who do not report rhinitis of a 'mixed' etiology (i.e., with both allergic and non-allergic triggers) (Martin et al., 2013).

Perhaps most relevant for nurse practitioners and other providers, our findings also suggest headache symptoms among disadvantaged women may be poorly controlled and/or managed within the current health care system. Only $56 \%$ of adults who meet migraine criteria are aware their headaches are migraines (Bigal, Borucho, Serrano, \& Lipton, 2009), and only $26 \%$ of adults with significant migraine-related functional impairment have accessed care, received an appropriate diagnosis and/or migraine-specific treatment (Lipton et al., 2013). Current evidence-based guidelines recommend a stratified treatment approach for migraine, which urges clinicians to take into account several factors in treatment decision-making. These include pain intensity, degree of disability, and comorbid conditions, among others (National Headache Foundation, 2013). While recommended firstline medications for acute migraine can include simple analgesics such as aspirin, acetaminophen, and/or NSAIDS, triptans are considered the mainstay of migraine treatment unless contraindicated. Based on 2009 ambulatory care data, when prescription medications are given to treat migraine, $82 \%$ are for triptans (Smitherman, Burch, Sheikh, \& Loder, 2013). Although migraine status was not definitively established in the current sample, the pattern of medication use in this sample differs somewhat from other published findings of acute treatment for episodic migraine in the adult population, where OTC preparations were used by $68 \%, 32 \%$ were using no medications, $19.3 \%$ were using migraine-specific medications, and opiates or butalbital-containing compounds were used by $11.1 \%$ and $6 \%$, respectively (Bigal et al., 2009). Given the higher likelihood that many in our sample would meet migraine or probable migraine criteria, very few women in our sample were using triptans $(7.6 \%)$ compared to triptan use among adults with migraine in the general population (19.3\%) (Bigal et al., 2009).

Compared to the $7.3 \%$ of adults with migraine in the ongoing American Migraine Prevalence and Prevention (AMPP) study cohort who reported an ED visit in the prior year for headache (Smitherman et al., 2013), the $36.6 \%$ of women in our study who visited the $\mathrm{ED}$ for headache in the prior year was remarkably high. This is particularly so when the vast majority (91\%) who made an ED visit also reported their headaches were present for two or more years. There are several factors that could account for higher ED use in this group. First, although all women in the study were receiving Medicaid at the time these data were collected, many probably lacked health insurance at some point in the prior year, which may have limited primary care access. Second, it is equally likely the result of poor overall 
headache symptom management by the women themselves, their health care providers, or both. Among women who were taking migraine-specific medications (triptans or ergots) for headache, more than $50 \%$ had an ED visit in the prior year specifically due to their headache symptoms.

For nurse practitioners who provide care to this population, these findings suggest a need for more extensive headache symptom evaluation and screening, education on symptom management, and regular monitoring of treatment efficacy. Given the association of headache with job loss in this group - particularly in the context of a greater number of CHCs generally and when allergies are also present - providers should be explicitly asking women how their headaches may or may not be interfering with functioning at work and elsewhere. This conversation should include what symptom triggers may be precipitated by their employment settings or roles, and what their symptom management strategies are when headaches occur on work days and/or while at work. Given the need for many disadvantaged women to provide economic support for their families and the characteristics of the jobs held by this group, ensuring headache symptoms are not contributing to health-related job loss is imperative.

Finally, further research is needed. While studies have shown lower-income groups have a higher prevalence of migraine and more functional impairment from migraine, little is known about how disadvantaged women are managing their headache symptoms, how their choice of management may be affecting symptoms related to other comorbid conditions, and how their management strategies may be impacting their functioning in select roles (particularly with regard to employment). Studies have shown the national impact that headaches have in terms of lost productivity and wages, and clinical trials have demonstrated an estimated 31-35\% of the productivity loss can be reversed solely with the use of triptans (Adelman, Sharfman, \& Johnson, 1996; Mushet, Miller, Clements, Pait, \& Gutterman, 1996). Generally, however, studies of the symptom management strategies used by disadvantaged women - and how these might affect role functioning and work performance - are lacking. Additional research in this area is needed to determine whether the development of interventions that are more culturally tailored to the daily realities of the employment conditions and demands of this group.

\section{Conclusions}

The presence of headache and a higher number of CHCs increases the probability of healthrelated job loss for disadvantaged women. This risk is exacerbated when allergies co-occur, and may be increased with higher numbers of CHCs and for women with lower levels of education. The care nurse practitioners provide is often focused on underserved populations, as well as prevention and symptom management. As such, nurse practitioners are well suited for not only tailoring pharmacological headache therapy to individual patient characteristics, but also for providing the additional education, cognitive-behavioral therapies, and/or symptom monitoring that disadvantaged women may need for optimal headache symptom management. This is essential to minimize not only headache-related disability, but also to ensure headaches do not contribute to job loss and further economic instability for this vulnerable population. 


\section{Acknowledgments}

The authors disclosed receipt of the following financial support for the research and/or authorship of this article: The first author received a grant from the National Institutes of Health and the National Institute of Nursing Research (NIH/NINR Grant \# 5R01NR009406), which funded the research. The contents of this article are solely the responsibility of the authors and do not necessarily represent the official views of the NIH or the NINR.

\section{References}

Adelman JU, Sharfman M, Johnson R. Impact of oral sumatriptan on workplace productivity, healthrelated quality of life, healthcare use, and patient satisfaction with medication in nurses with migraine. American Journal of Managed Care. 1996; 2(11):1407-1416.

Bigal ME, Bordini CA, Speciali JG. Etiology and distribution of headaches in two Brazilian primary care units. Headache. 2000; 40(3):241-247. [PubMed: 10759928]

Bigal ME, Borucho S, Serrano D, Lipton RB. The acute treatment of episodic and chronic migraine in the USA. Cephalalgia. 2009; 29(8):891-897. doi: 10.1111/j.1468-2982.2008.01819.x. [PubMed: 19222509]

Buse DC, Loder EW, Gorman JA, Stewart WF, Reed ML, Fanning KM, Serrano D, Lipton RB. Sex differences in the prevalence, symptoms, and associated features of migraine, probable migraine and other severe headache: results of the American Migraine Prevalence and Prevention (AMPP) Study. Headache. 2013; 53(8):1278-1299. doi: 10.1111/head.12150. [PubMed: 23808666]

First, MB.; Spitzer, RL.; Gibbon, M.; Williams, JBW. Structured Clinical Interview for DSM-IV-TR Axis I Disorders, Research Version. Biometrics Research, New York State Psychiatric Institute; New York: 2002.

Hainer BL, Matheson EM. Approach to acute headache in adults. [Review]. Am Fam Physician. 2013; 87(10):682-687. [PubMed: 23939446]

International Headache Society (IHS). The International Classification of Headache Disorders. 2nd Edition, 1st revision. Blackwell Publishing; Oxford: Oxford: May. 20052005 p. 232p. 232

Jensen R, Stovner LJ. Epidemiology and comorbidity of headache. [Review]. Lancet Neurol. 2008; 7(4):354-361. doi: 10.1016/S1474-4422(08)70062-0. [PubMed: 18339350]

Kessler RC, Shahly V, Stang PE, Lane MC. The associations of migraines and other headaches with work performance: Results from the National Comorbidity Survey Replication (NCS-R). Cephalalgia. 2010; 30(6):722-734. doi: 10.1177/0333102410363766. [PubMed: 20511212]

$\mathrm{Ku}$ M, Silverman B, Prifti N, Ying W, Persaud Y, Schneider A. Prevalence of migraine headaches in patients with allergic rhinitis. Annals of Allergy, Asthma, \& Immunology. 2006; 97(2):226-230. doi: 10.1016/S1081-1206(10)60018-X.

Lipton RB, Serrano D, Holland S, Fanning KM, Reed ML, Buse DC. Barriers to the diagnosis and treatment of migraine: effects of sex, income, and headache features. Headache. 2013; 53(1):8192. doi: 10.1111/j.1526-4610.2012.02265.x. [PubMed: 23078241]

Lipton RB, Stewart WF, Diamond S, Diamond ML, Reed M. Prevalence and burden of migraine in the United States: data from the American Migraine Study II. Headache. 2001; 41(7):646-657. [PubMed: 11554952]

Martin VT, Fanning KM, Serrano D, Buse DC, Reed ML, Bernstein JA, Lipton RB. Chronic rhinitis and its association with headache frequency and disability in persons with migraine: Results of the American Migraine Prevalence and Prevention (AMPP) Study. Cephalalgia. 2013 doi: $10.1177 / 0333102413512031$.

Mitchell, MN. Interpreting and Visualizing Regression Models Using Stata. StatCorp, LP.; College Station, TX: 2012.

Mushet GR, Miller D, Clements B, Pait G, Gutterman DL. Impact of sumatriptan on workplace productivity, nonwork activities, and health-related quality of life among hospital employees with migraine. Headache. 1996; 36(3):137-143. [PubMed: 8984084]

National Headache Foundation. Standards of Care for Headache Diagnosis and Treatment. National Headache Foundation; 2013. http://www.headaches.org/content/standards-care-headachediagnosis-and-treatment 
Singh-Manoux A, Ferrie JE, Chandola T, Marmot M. Socioeconomic trajectories across the life course and health outcomes in midlife: evidence for the accumulation hypothesis? International Journal of Epidemiology. 2004; 33:1072-1079. [PubMed: 15256527]

Smitherman TA, Burch R, Sheikh H, Loder E. The prevalence, impact, and treatment of migraine and severe headaches in the United States: a review of statistics from national surveillance studies. Headache. 2013; 53(3):427-436. doi: 10.1111/head.12074. [PubMed: 23470015]

StataCorp, LP. (Version 13.0 ed.).Stata Data Analysis and Statistical Software. 2013. www.stata.com

Stewart WF, Roy J, Lipton RB. Migraine prevalence, socioeconomic status, and social causation. Neurology. 2013; 81(11):948-955. doi: 10.1212/WNL.0b013e3182a43b32. [PubMed: 23990405]

Stovner L, Hagen K, Jensen R, Katsarava Z, Lipton R, Scher A, Steiner T, Zwart JA. The global burden of headache: a documentation of headache prevalence and disability worldwide. Cephalalgia. 2007; 27(3):193-210. doi: 10.1111/j.1468-2982.2007.01288.x. [PubMed: 17381554]

Strully KW. Job loss and health in the U.S. labor market. Demography. 2009; 46(2):221-246. doi: 10.1353/dem.0.0050. [PubMed: 21305391]

World Health Organization. Fact Sheet. World Health Organization; Oct. 2012 Headache Disorders Fact Sheet. http://www.who.int/mediacentre/factsheets/fs277/en/ 


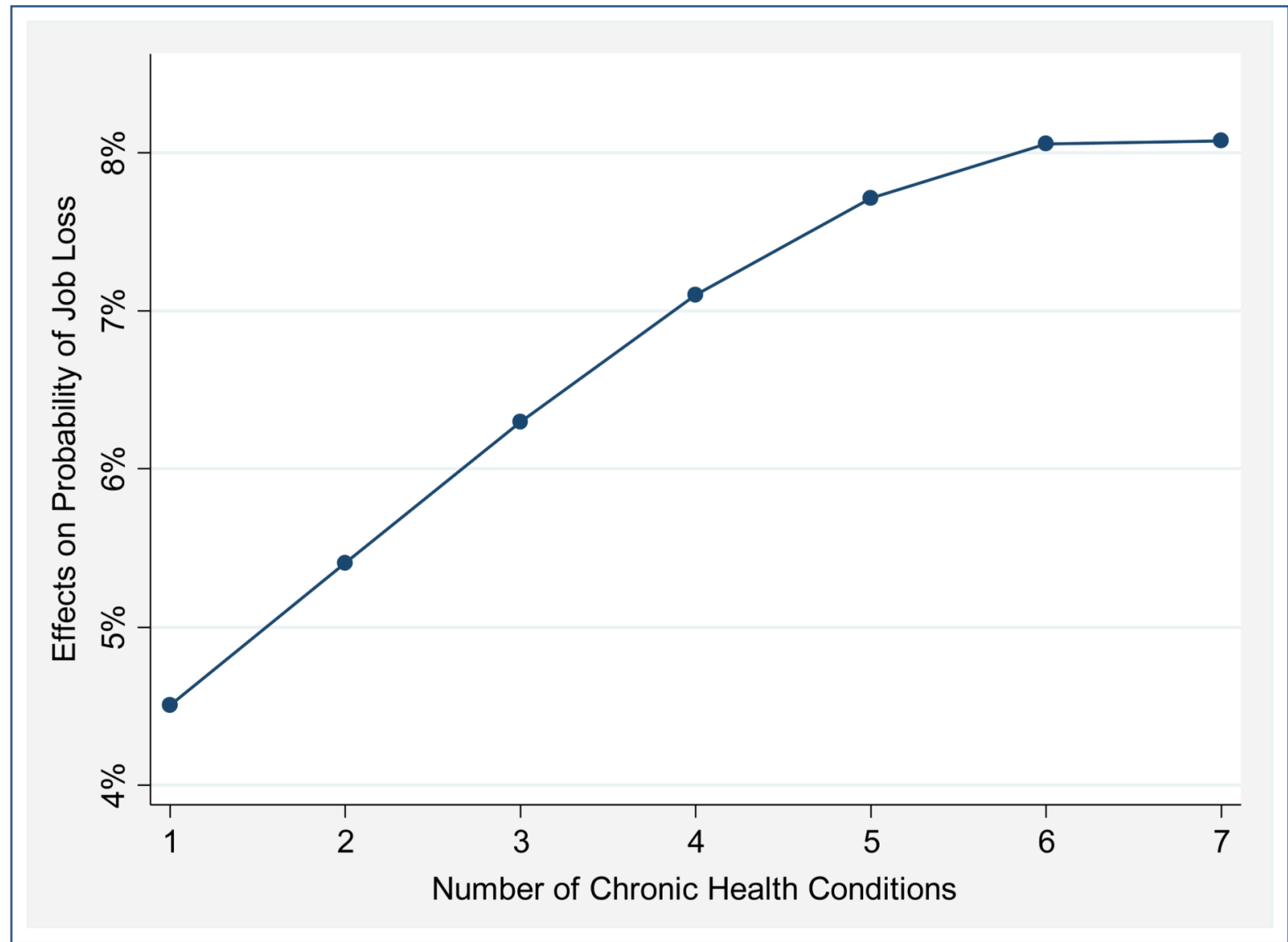

*Job loss is specific to health-related job loss in prior year. Average marginal effects depict the marginal, or "additional," or "unique" effect of having headache as a chronic health condition on the probability of healthrelated job loss in the prior year.

Figure 1.

Marginal Effects of Headache on Job Loss, by Number of Chronic Health Conditions $(\mathrm{CHCs})^{*}$ 


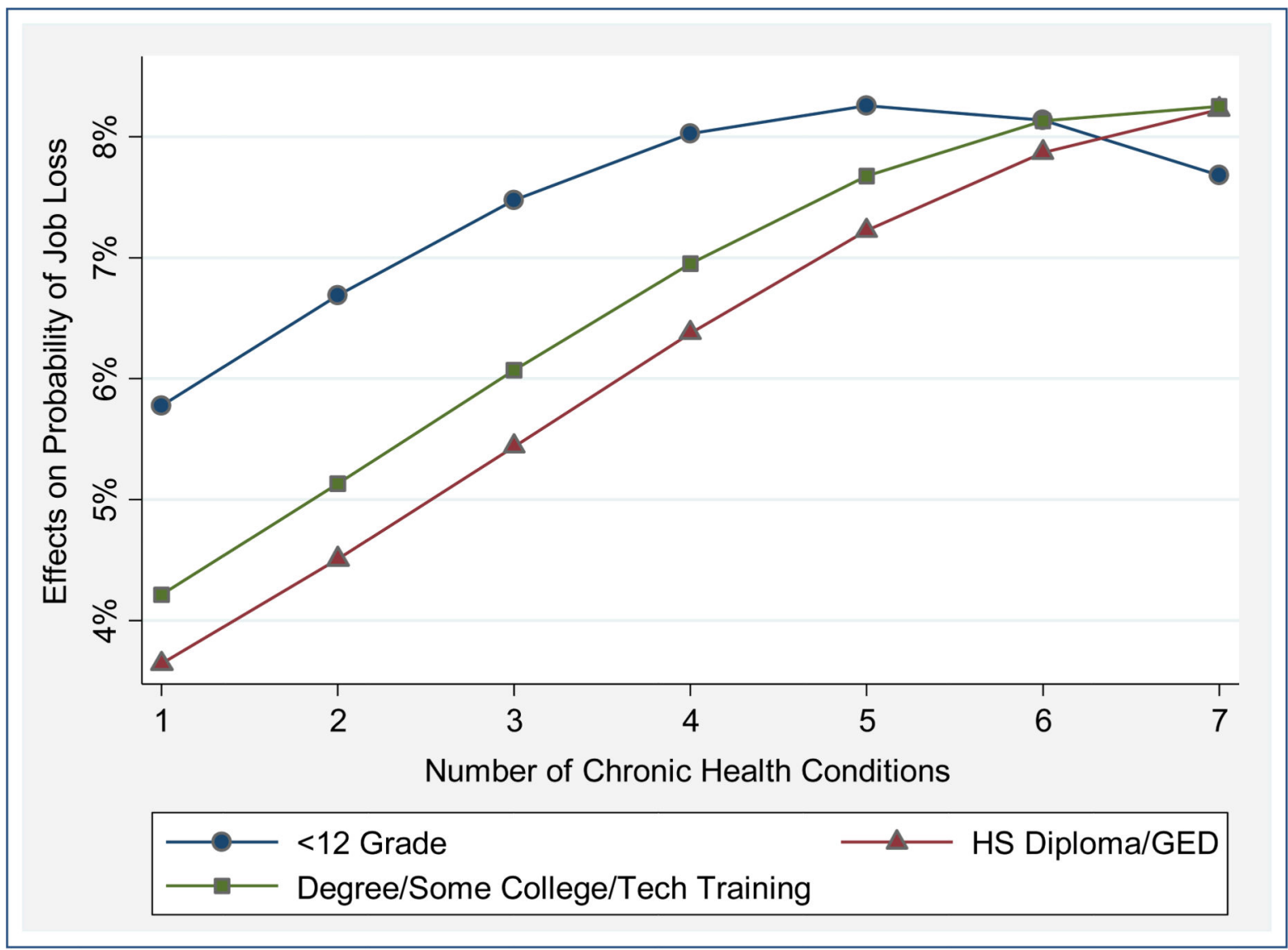

*Job loss is specific to health-related job loss in prior year. Average marginal effects depict the marginal, or "additional," or "unique" effect of having headache as a chronic health condition on the probability of health-related job loss in the prior year, stratified by education level.

Figure 2.

Marginal Effects of Headache on Job Loss, by Educational Level and Number of Chronic Health Conditions (CHCs)* 


\section{Table 1}

Sociodemographic Characteristics, at Baseline $(\mathrm{n}=432)$

\begin{tabular}{|lc|}
\hline Characteristic & \% or Mean (SD) \\
\hline Age & $29.8(8.2)$ \\
Race & \\
Black & $56.3 \%$ \\
White & $39.4 \%$ \\
Other & $4.4 \%$ \\
Ethnicity: Hispanic & $4.6 \%$ \\
Education level & \\
$<12^{\text {th }}$ Grade & $32.4 \%$ \\
High school diploma or GED & $27.8 \%$ \\
Some college or technical training & $38 \%$ \\
College degree ( $\geq$ associate degree) & $1.8 \%$ \\
Total household income (per month, in dollars) & $656.6(434.4)$ \\
Number of jobs in past year & \\
0 & $19.4 \%$ \\
$1-2$ & $61.8 \%$ \\
$3-4$ & $13.7 \%$ \\
$\geq 5$ & $5.1 \%$ \\
Number of jobs left in past year due to health & \\
0 & \\
$\geq 1$ & \\
Number of chronic health conditions & $3.8(2.3)$ \\
\hline
\end{tabular}

* Includes income per month from food stamps, TANF, work-related income, and income received from others (e.g., family, friends, or partners) on a regular basis. 
Table 2

Odds of Reporting Leaving Job due to Health within Past Year by Headache as a Reported Chronic Health Condition (CHC), Adjusted for Select Covariates $(\mathrm{n}=348)^{* *}$

\begin{tabular}{|c|c|c|c|c|c|}
\hline Variables & Model 1 & Model 2 & Model 3 & Model 4 & Model 5 \\
\hline \multicolumn{6}{|l|}{ Independent Variable of Interest } \\
\hline \multicolumn{6}{|l|}{$\begin{array}{l}\text { Headache Reported as Chronic Health } \\
\text { Condition }\end{array}$} \\
\hline No & Ref. & Ref. & Ref. & Ref. & Ref. \\
\hline Yes & $1.99^{*}(1.25,3.17)$ & $2.00 *(1.26,3.19)$ & $1.98^{*}(1.24,3.16)$ & $1.99^{*}(1.24,3.20)$ & $1.40(.84,2.31)$ \\
\hline \multicolumn{6}{|l|}{ Select Covariates, Added Sequentially } \\
\hline Age & & $1.01(.98,1.03)$ & $1.01(.98,1.04)$ & $1.01(.98,1.04)$ & $.99(.95,1.02)$ \\
\hline \multicolumn{6}{|l|}{ Race } \\
\hline White & & & Ref. & Ref. & Ref. \\
\hline Black & & & $1.03(.64,1.66)$ & $1.00(.62,1.63)$ & $1.39(.83,2.35)$ \\
\hline Other & & & $1.49(.48,4.64)$ & $1.55(.49,4.87)$ & $1.26(.37,4.32)$ \\
\hline \multicolumn{6}{|l|}{ Education } \\
\hline$<12^{\text {th }}$ grade & & & & Ref. & Ref. \\
\hline High school/GED & & & & $.51^{*}(.28, .95)$ & $.51^{*}(.27, .95)$ \\
\hline Some college/college degree & & & & $.79(.45,1.36)$ & $.66(.37,1.19)$ \\
\hline $\begin{array}{l}\text { Number of chronic health conditions } \\
\text { (total) }\end{array}$ & & & & & $1.35 *(1.19,1.53)$ \\
\hline Constant & .317 & .267 & .258 & .353 & .214 \\
\hline
\end{tabular}




\section{Table 3}

Chronic Health Conditions (CHCs) that Significantly Increase the Odds of Reporting Leaving Job due to Health within Past Year $(\mathrm{n}=348)^{* *}$

\begin{tabular}{lcccc}
\hline & OR & SE & P & $(\mathbf{9 5 \%}$ CI $)$ \\
\hline Chronic Health Conditions & & & & \\
\multicolumn{1}{l}{ Retained $^{*}$} & & & & \\
$\quad$ Headache & 2.01 & .49 & .004 & $(1.25,3.23)$ \\
Allergies & 1.78 & .43 & .02 & $(1.11,2.86)$ \\
& Not Retained \\
Back pain & & & & \\
Depression & --- & --- & --- & --- \\
Anxiety & --- & --- & --- & --- \\
\hline
\end{tabular}

*Adjusted for age, race, and educational level, significance criteria for retention set at $\mathrm{p}<.20$.

**

Three of the five most frequently reported chronic health conditions not retained in the model $(p \geq 20)$ 